\title{
Concurrent counting of two and three events in a serial anticipation paradigm
}

\author{
RICHARD A. BURNS and REBECCA E. SANDERS \\ Georgia Southwestern College, Americus, Georgia
}

\begin{abstract}
Rats were runway trained with four different series of reward events for the purpose of determining if two different number cues (two and three) could be adequately employed to correctly anticipate a terminal nonreward in the series. In two of the series, two rewarded runs always occurred prior to terminal nonreward; one of these series always began with a nonrewarded run, NRRN, and the other did not, RRN. For two other series, three rewarded runs predicted terminal nonreward, NRRRN and RRRN. Each rat experienced each of the four series once a day for 26 days. The interval between runs within a series was about $20 \mathrm{sec}$, the interval between series was about $15 \mathrm{~min}$, and the order in which the series were presented was randomized daily. Distinctive floor cues (smooth/black or rough/white, balanced over animals) signaled two-count and three-count series. Our animals correctly anticipated (ran slowly to) the terminal nonreward of all four series. On Day 27, an initial nonrewarded run was added to the two series that had been initiated by nonreward (NNRRN and NNRRRN). This addition produced a disruption in terminalevent anticipation that lasted for only 1 training day. In all, the results imply that the animals were concurrently counting both two and three rewarded events, and it is further suggested that counting plays a prominent role in the rat's serial learning.
\end{abstract}

Davis and Memmott (1982), in a review of the literature pertinent to counting in animals, concluded that animals can be trained in such a way that number cues control their behavior, but that counting is an unprepared or contraprepared (Seligman, 1970) activity. The first conclusion was based on an impressive number of reports in the literature that suggested the operation of counting processes. The second conclusion was based on trials-tocriterion judgments of difficulty in those reports and on problems with fashioning a tale of evolutionary significance for counting to conjoin the results and Seligman's ideas.

Capaldi and Miller (in press a), on the other hand, recently decided that counting processes play a prominent role in instrumental learning situations, and that rats not only count, but count routinely, in at least their instrumental learning. In the seven new experiments they reported, rats were runway trained under conditions in which correct anticipation of (slower running to) a nonreward event that terminated a series of other events depended upon their counting two (Experiments 1, 2, 3, 5, 6, and 7) or three (Experiments 4 and 5) preceding reward events. Correct anticipation of terminal nonreward was clear in all of the investigations in which number was a reliable predictor.

In the present experiment, we used the apparently more sensitive methods of Capaldi and Miller (in press a) to look for evidence of the rat's ability to concurrently count

This work was supported by a grant to the first author from the Charles L. Mix Foundation. Address correspondence to the first author, Department of Psychology, Georgia Southwestern College, Americus, GA 31709. both two and three events. There were two primary reasons for doing this: (1) We are inclined to agree that counting is routinely employed by rats and that there are other instrumental situations, which will be discussed later, that may involve as yet unsuspected counting of two and three events. (2) Many of the counting investigations reported so far have required animals to respond to a single number cue rather than to multiple (in this case, two) number cues, but Capaldi and Miller's (in press b) Experiment 5 suggests that multiple counting does occur in serial learning.

\section{METHOD}

\section{Subjects}

The subjects were 4 naive male rats purchased from the Holtzman Company, Madison, WI. The animals were about 90 days old at the beginning of training.

\section{Apparatus}

The L-shaped wooden runway ( $233.7 \mathrm{~cm}$ long) we used was covered with hinged clear plastic and equipped with manually operated start and retrace doors. Measures of total running time were read from a Standard Electric timer, which was activated when the start door opened and was stopped when the rat passed a photocell mounted $195.6 \mathrm{~cm}$ beyond the start door. A right turn $182.9 \mathrm{~cm}$ beyond the start door marked entry into the goal area, which contained a pellet dish fashioned from a handleless teaspoon. The entire runway was painted black, but a floor panel made of aluminum truck-bumper stock with a raised diamond pattern was painted white and hinge mounted so that the floor of the runway could be easily made rough and white or smooth and black.

\section{Procedure}

All animals were housed in individual living cages when they arrived. The cages were located in the room that contained the runway. For the first 3 days, unrestricted access to food and water was allowed, and body weights were recorded, following a 15 - to 20 -min exercise period 
on a walled table. A food-deprivation procedure was then begun and maintained throughout the experiment. The procedure reduced animals gradually to $85 \%$ of their free-feeding body weights and maintained them at that level by controlling the ration given at the end of daily sessions.

There were then 4 days of goal-area placements. Each rat was placed in the goal area five times each day with the retrace door closed and the pellet dish baited with $10.045-\mathrm{g}$ Noyes pellets. Whether the floor was white and rough or black and smooth alternated from one placement to the next. The rats were removed from the goal area after $5 \mathrm{~min}$ (this occurred only twice) or after the reward had been consumed. By the end of goal training, all rats were consuming the 10 pellets in less than $1 \mathrm{~min}$.

During the 26 days of primary experimental training, all rats were run each day with each of four reward series. In two of the series, two rewarded runs always preceded a nonrewarded run; and one of these two series, however, began with a nonrewarded run. These series can be represented as NRRN and RRN. In the remaining two series, three rewarded runs always preceded a nonrewarded run, RRRN, and one of the series was initiated by a nonrewarded run, NRRRN. For a random half of the animals, the NRRN/RRN series always occurred with the black, smooth floor and the NRRRN/RRRN series occurred with the white, rough floor. The remaining half had the cues reversed. The order in which a rat experienced each of the four series was determined randomly each day, as was the order in which the animals were run. A given animal was run on all runs within a series successively, making the interval between the runs about $20 \mathrm{sec}$. Another series was initiated only after all other animals had run their series, making an interval between series of about $15 \mathrm{~min}$.

A run began with the opening of the start door about $2 \mathrm{sec}$ after the rat was placed in the start area, and it terminated when the rat had consumed the reward (10 pellets) or after $30 \mathrm{sec}$ in the unbaited goal area on nonrewarded runs. After each day's training, all 4 animals were placed on the exercise table for about $45 \mathrm{~min}$, and then weighed and fed. Between runs and series, the animals stayed in the living cage with water available.

After asymptotic performance had been established (after 26 days), an additional nonrewarded run was inserted in the two series that were initiated by nonrewarded runs, and training on the modified series, NNRRN/RRN and NNRRRN/RRRN, continued for 2 days.

\section{RESULTS}

All run times were transformed $[x=\log (x)]$ for statistical analysis. The average times for the runs within each of the four series are plotted in Figure 1. The terminal nonrewarded run is always shown as Run 5. Our statisti-

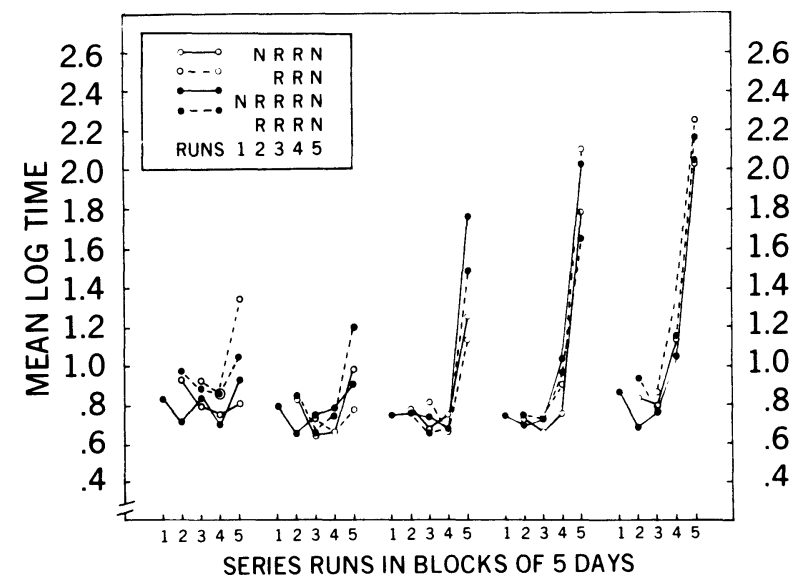

Figure 1. Mean transformed running times on each run of the four series in blocks of 5 days. The terminal run is noted as Run 5 irrespective of the actual number of runs in the series.

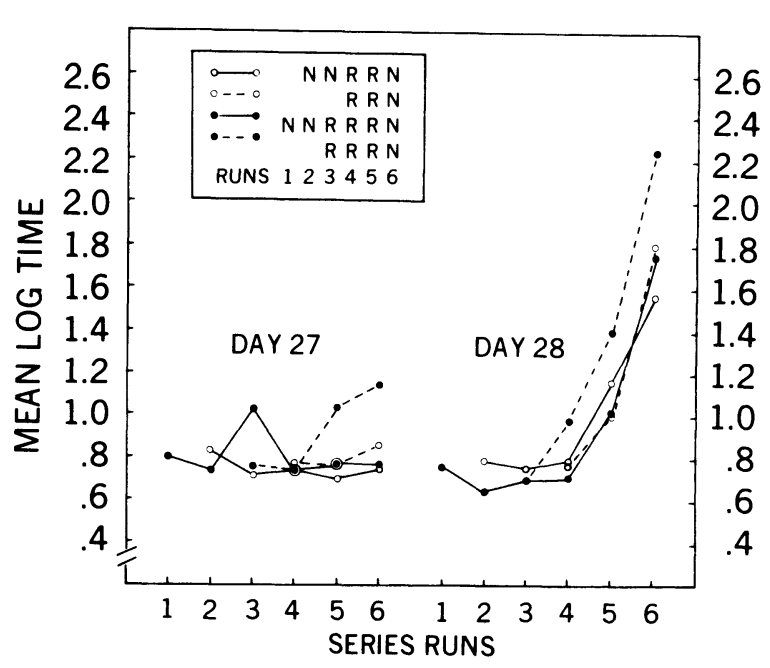

Figure 2. Mean transformed running times on the 2 days with the additional initial $\mathbf{N}$ event.

cal strategy was to examine each dayblock separately with analyses of variance. The Bonferroni $t$ statistic $(k=10$, $d f=8$ ) was used to evaluate main effect means for the run variable. All results are reported as significant if $p<.05$. It is clear that the rats developed correct anticipation of (slower approach to) the terminal nonreward event of the series. As early as the second dayblock, the animals were running significantly slower on the terminal nonrewarded run (Run 5) of the series $[F(4,8)=23.27]$. On the third dayblock, approach to the terminal nonreward was slower than on all other runs except Run $3[F(4,8)=10.98]$, and on the fourth and fifth dayblocks, running was slower on Run 5 than on all other runs, each of which did not differ from the other $[F(4,8)=18.17 ; F(4,8)=87.93]$. There was no evidence in these analyses that anticipation was any different among the different series.

The addition of an initial nonrewarded run on Day 27 simply eliminated the discriminations that were so clear before the addition, with the possible exception of those in the RRRN series. The exception is indicated by a significant runs $\times$ series interaction $[F(9,18)=3.06]$.

Figure 2 shows average times on each of the six series runs for Days 27 and 28. The disruption caused by the added nonreward event was short-lived. Analysis of Day 28 showed, once again, that the terminal nonreward was correctly anticipated in all series $[F(5,10)=21.43$; $k=14, d f=10]$.

\section{DISCUSSION}

There was clear evidence that these rats came to anticipate the terminal nonreward element of every series with which they had experience. In two of the series (NRRN and RRN), two rewarded events reliably predicted the terminal nonreward, and in the other two series (NRRRN and RRRN), three rewarded events predicted nonreward. These findings are in essential agreement with the results between subjects obtained by Capaldi and Miller (in press a), and they extend those findings by showing that anticipation, which is apparently based on two and three predictive events, can be obtained within subjects under the differential control of additional cues that were initially nonnumerical. 
Some interesting differences between our findings and those of Capaldi and Miller (in press a) are worth mentioning. In their Experiment 4, for instance, rats were trained on the series NRRRN/RRRN, a problem that required counting three reward events. Animals in that experiment ran slower to the terminal nonreward than to any other event in both series, but they also ran slower on the rewarded run that preceded the terminal element than they did on the other rewarded runs. Because this slower approach to the last reward in the series did not appear in their other experiments in which the rats had to count two rather than three rewarded events and because the slower approach appeared on the last rewarded run of both the NRRRN and the RRRN series, Capaldi and Miller (in press a) concluded that it was not caused by remote anticipation of the terminal nonreward. Instead they proposed that rats might have had some difficulty counting three as opposed to two events.

In the present set of results, there was a tendency, evident especially on the last dayblock of primary training, to run slower on the last rewarded run, but there was no indication that the tendency was any different on series that involved two and three events. Counting was equally correct on both kinds of series, which suggests that the hint of slower running prior to nonreward was mediated by anticipation rather than by count confusion, and it may be that the within-subjects procedure facilitates the discriminations of number cues.

One of the many roles that counting may play in instrumental learning involves serial anticipation. Counting is a plausible basis for information pertaining to the ordinal position of an event in a regularly presented series. Associations between the ordinal position of an event and instrumental behavior may mediate some serial learning in animals (e.g., Burns, Wiley, \& Payne, 1986; Terrace, 1986). Consider an experiment reported by Capaldi, Verry, and Nawrocki (1982). Rats were given two series of rewards in a runway each day in such a way that the order in which animals ran the series could not have reliably predicted any particular series. One pair of series studied was 20-0-20 and 2-0, where each number refers to the number of $.045-\mathrm{g}$ pellets given as reward. In these series, the rats learned to run generally faster on rewarded than on nonrewarded runs, and they showed what has come to be called interevent anticipation (Capaldi, Nawrocki, \& Verry, 1983): slower running on Run 2 of the 2-0 series than on Run 2 of the 20-0-20 series.

This particular serial learning result seems best explained as the consequence of remote and adjacent associations among the sensory representations of items in the series list as opposed to associations between the ordinal position of an item and instrumental behavior, because the memory of the initial reward amount in each series was the only reliable series cue (see Capaldi \& Miller, in press b, for an elaboration of this idea). If memory associations were necessary to discriminate the two series, they might also have been used to anticipate the items within a series. However, it is possible that the initial reward served as noth- ing more than a series cue setting a discrimination based instead on counting and ordinal-position information; a 20-pellet reward signaled a threeposition series, and a two-pellet reward signaled a two-position series. The results of the present experiment add some credibility to this position hypothesis because they show that rats can concurrently count two and three if the counting situations are differentially cued.

It is, in fact, possible that in the series NRRN and RRN studied here, for example, the initial event in the series served as a series cue that signaled different numbers of events leading to terminal nonreward; initial nonreward signaled a four-event series and initial reward signaled a threeevent series. The addition of an extra initial nonreward event on Day 27 of the present experiment produced only a short-lived disruption in the correct anticipation of terminal nonreward, a fact that works against this view. A better reckoning, of the present results at least, is that the counting of reward events alone was the basis of the rat's anticipation.

\section{REFERENCES}

Burns, R. A., Wiley, L. P., \& Payne, T. L. (1986). Temporal cuing of runs in series of reward events reduces interevent anticipation. Animal Learning \& Behavior, 14, 190-196.

CAPALDI, E. J., \& Miller, D. J. (in press a). Counting in rats: Its functional significance and the independent cognitive processes which comprise it. Journal of Experimental Psychology: Animal Behavior Processes.

CaPAldi, E. J., \& Miller, D. J. (in press b). The rat's simultaneous anticipation of remote events and current events can be sustained by event memories alone. Animal Learning \& Behavior.

Capaldi, E. J., NAWrocki, T. M., \& VerRY, D. R. (1983). The nature of anticipation: An inter- and intraevent process. Animal Learning \& Behavior, 11, 193-198.

Capaldi, E. J., Verry, D. R., \& NaWrocki, T. M. (1982). Multiple hedonic memory: Memory for more than one hedonic event in the rat. Animal Learning \& Behavior, 10, 351-357.

Davis, H., \& Memmotт, J. (1982). Counting behavior in animals: A critical evaluation. Psychological Bulletin, 92, 547-591.

Seligman, M. E. P. (1970). On the generality of the laws of learning. Psychological Review, 77, 406-418.

TERRACE, H. S. (1986). A nonverbal organism's knowledge of ordinal position in a serial learning task. Journal of Experimental Psychology: Animal Behavior Processes, 12, 203-214.

(Manuscript received for publication May 13, 1987.) 